\title{
Modelling of the surface magnetic field in neutron stars: Application to radio pulsars
}

\author{
J. A. Gil ${ }^{1}$, G. I. Melikidze ${ }^{1,2}$, and D. Mitra ${ }^{3}$ \\ 1 Institute of Astronomy, University of Zielona Góra, Lubuska 2, 65-265, Zielona Góra, Poland \\ 2 Center for Plasma Astrophysics, Abastumani Astrophysical Observatory, Al.Kazbegi ave. 2a, Tbilisi 380060, \\ Georgia \\ 3 Max-Planck Institute for Radioastronomy, Auf dem Hügel 69, 53121 Bonn, Germany
}

Received 27 November 2001 / Accepted 25 March 2002

\begin{abstract}
We propose a vacuum gap (VG) model which can be applied uniformly for normal and high-magneticfield pulsars. The model requires a strong and non-dipolar surface magnetic field near the pulsar polar cap. We assume that the actual surface magnetic field $B_{\mathrm{s}}$ in pulsars results from the superposition of a global dipole field $B_{\mathrm{d}}$ and a crust-anchored small scale magnetic anomaly $B_{\mathrm{m}}$. We provide a numerical formalism for modelling such structures of the surface magnetic field and explore it within the framework of the VG model, which requires strong surface fields $B_{\mathrm{s}} \gtrsim 10^{13} \mathrm{G}$. Thus, in order to increase the resultant surface field to values exceeding $10^{13} \mathrm{G}$, in low magnetic field pulsars with $B_{\mathrm{d}} \ll 10^{13} \mathrm{G}$ it is required that $B_{\mathrm{m}} \gg B_{\mathrm{d}}$, with the same polarities (orientations) of $B_{\mathrm{d}}$ and $B_{\mathrm{m}}$. However, if the polarities are opposite, the resultant surface field can be lower than the dipolar surface component inferred from the pulsar spin-down. We propose that high-magnetic-field pulsars (HBPs) with the inferred global dipole field $B_{\mathrm{d}}$ exceeding the so-called photon splitting threshold $B_{\mathrm{cr}} \sim 4 \times 10^{13} \mathrm{G}$, can generate observable radio emission "against the odds", provided that the surface dipolar magnetic field $B_{\mathrm{d}}$ is reduced below $B_{\mathrm{cr}}$ by the magnetic anomaly $B_{\mathrm{m}}$ of the right strength and polarity. We find that effective reduction is possible if the values of $B_{\mathrm{d}}$ and $B_{\mathrm{m}}$ are of the same order of magnitude, which would be expected in HBPs with $B_{\mathrm{d}}>B_{\mathrm{cr}}$. The proposed VG model of radio emission from HBPs, in which pair production occurs directly above the polar cap, is an alternative to the recently proposed lengthened space-charge-limited-flow (SCLF) model, in which the pair formation front is located at relatively high altitudes, where the dipole field is degraded below $B_{\mathrm{cr}}$. Our model allows high $B_{\mathrm{d}}$ radio-loud pulsars not only just above $B_{\mathrm{cr}}$ but even above $2 \times 10^{14} \mathrm{G}$, which is the upper limit for HBPs within the lengthened SCLF model.
\end{abstract}

Key words. pulsars: general

\section{Introduction}

There are two important conclusions that can be drawn from the radio emission properties of pulsars. Firstly the radio emission is thought to arise at an altitude $r_{\mathrm{em}}$ from the center of the neutron star, where $r_{\mathrm{em}}$ is of the order of several stellar radii, $R=10^{6} \mathrm{~cm}$ (e.g. Kijak \& Gil 1997, 1998, and references therein). Secondly the regions from where the radio emission arises are consistent with a purely dipolar magnetic field (Radhakrishnan \& Cooke 1969). However the structure of the magnetic field at the surface of the neutron star is largely unknown. Strong non-dipolar surface magnetic fields have long been thought to play an important role in the radio emission of pulsars. For example, in order to sustain pair production in vacuum gaps, the Ruderman \& Sutherland (RS75) model implicitly assumed that the radius of curvature of field lines above the polar cap should be about $10^{6} \mathrm{~cm}$,

Send offprint requests to: J. A. Gil, e-mail: jag@astro.ca.wsp.zgora.pl which is 100 times smaller than that expected from a global dipolar magnetic field, thus indicating the presence of non-dipolar components. It is believed that thermal X-rays from pulsars are a good diagnostic tool to infer the structure of the surface magnetic field. Soft X-ray observations of pulsars show non-uniform surface temperatures which can be attributed to small scale magnetic anomalies on the pulsar polar cap (e.g. Page \& Sarmiento 1990; Bulik et al. 1992, 1995). Several similar arguments in favour of the non-dipolar nature of the surface magnetic field can also be found in Becker \& Trümper (1997); Cheng et al. (1998); Rudak \& Dyks (1999); Cheng \& Zhang (1999); Thompson \& Duncan (1995, 1996), Murakami et al. (1999), and Tauris \& Konar (2001). Also several theoretical studies concerning the formation and evolution of non-dipolar magnetic fields in neutron stars are found in the literature (e.g. Blandford et al. 1983; Krolik 1991; Ruderman 1991; Arons 1993; Chen \& Ruderman 1993; Geppert \& Urpin 1994; Mitra et al. 1999). 
Woltjer (1964) proposed that the magnetic field in neutron stars results from the fossil field of the progenitor star which is amplified during the collapse stage and remains anchored in the superfluid core of the neutron star. It was also noted by several authors that shortly after or during the collapse of the neutron star magnetic fields can be generated in the outermost crust (e.g. by a mechanism like thermomagnetic instabilities; Blandford et al. 1983). Urpin et al. (1986) showed that in the crustal model it is possible to form small-scale surface field anomalies with typical sizes of the order of 100 meters. Further, Gil \& Mitra (2001) demonstrated that such "sunspot"-like magnetic field structures on the polar cap surface help to sustain VG-driven radio emission of pulsars. In this paper we consider the scenario where the magnetic field on the neutron stars' surface is non-dipolar in nature which is due to superposition of the fossil field in the core and the crustal field structures. The crust gives rise to smallscale anomalies which can be modelled by a number of crust-anchored dipoles oriented in different directions (e.g. Blandford et al. 1983; Arons 1993). The superposition of global dipole and local anomaly is illustrated in Fig. 1, where for clarity of presentation only one local, crustassociated dipole is marked.

Formation of dense electron-positron pair plasma is essential for pulsar radiation, especially (but not only) at radio wavelengths. A purely quantum process for magnetic pair production $\gamma \rightarrow \mathrm{e}^{-} \mathrm{e}^{+}$is commonly invoked as a source of this plasma (e.g. Sturrock 1971; Ruderman \& Sutherland 1975). For superstrong magnetic fields close to the so-called quantum field $B_{\mathrm{q}}=4.4 \times 10^{13} \mathrm{G}$, the process of free $\mathrm{e}^{-} \mathrm{e}^{+}$pair production can be dominated by the phenomenon of photon splitting (Adler et al. 1970; Bialynicka \& Bialynicki et al. 1970; Baring \& Harding 1998) and/or bound positronium formation (Usov \& Melrose 1995, 1996). While the latter process can reduce the number of free pairs at magnetic fields $B \gtrsim 0.1 B_{\mathrm{q}}$ (e.g. Baring \& Harding 2001), the former can suppress the magnetic pair production at $B \gtrsim 10^{13} \mathrm{G}$ entirely, provided that photons which are polarized both parallel and perpendicular to the local magnetic field direction can split (e.g. Baring 2001; Baring \& Harding 2001). This assumption will be implicitly made throughout this paper. Under these circumstances one can roughly define a photon splitting critical line $B_{\mathrm{cr}} \sim B_{\mathrm{q}}$ and expect that there should be no radio pulsar above this line on the $B_{\mathrm{p}}-P$ diagram, where $B_{\mathrm{p}}=6.4 \times 10^{19}(P \dot{P})^{1 / 2} \mathrm{G}$ is the dipole surface magnetic field estimated at the pole from the pulsar period $P$ and its derivative $\dot{P}$ (Shapiro \& Teukolsky 1983; Usov \& Melrose 1996). This death-line is more illustrative than quantitative. In fact, a number of specific model-dependent death-lines separating radio-loud from radio-quiet pulsars are available in the literature (Baring \& Harding 1998, 2001; Zhang \& Harding 2000a, 2001). All these slightly period-dependent death-lines cluster around $B_{\mathrm{q}}$ on the $B_{\mathrm{p}}-P$ diagram, and hence the quantum critical field is conventionally treated as a threshold magnetic field above which pulsar radio emission ceases. In this paper we also use this terminology, bearing in mind that the photon splitting threshold realistically means a narrow range of magnetic fields around the critical quantum field $B_{\mathrm{q}} \sim 4 \times 10^{13} \mathrm{G}$, certainly above $10^{13} \mathrm{G}$ (see review by Baring 2001). For convenience, in all numerical examples presented in Figs. 2-6 and subsequent discussions we assume that the threshold magnetic field $B_{\mathrm{cr}}=B_{\mathrm{q}}$.

In order to produce the necessary dense electronpositron plasma, a high-voltage accelerating region has to exist near the polar cap of pulsars. Two models of such acceleration regions are available in the literature, namely: the stationary space charge limited flow (SCLF) model (Sharleman et al. 1978; Arons \& Sharleman 1979; Arons 1981) in which charged particles flow freely from the polar cap, and the highly non-stationary vacuum gap (VG) models (Ruderman \& Sutherland 1975; Cheng \& Ruderman 1977, 1980; Gil \& Mitra 2001) in which the free outflow of charged particles from the polar cap surface is strongly impeded. In the VG models the charged particles accelerate within a height scale of about the polar cap radius (i.e. $\sim 10^{4} \mathrm{~cm}$ ), due to a high potential drop across the gap, while in the SCLF models particles accelerate within a height scale of a stellar radius $\sim 10^{6} \mathrm{~cm}$, due to the potential drop resulting from the curvature of field lines and/or the inertia of outstreaming particles. In both models the free $\mathrm{e}^{-} \mathrm{e}^{+}$pairs are created if the kinematic threshold $\varepsilon_{\gamma} \cdot \sin \theta_{\mathrm{t}}=2 m c^{2}$ is reached or exceeded and the local magnetic field is lower than the photon splitting threshold $B \sim B_{\mathrm{cr}}$, where $\varepsilon_{\gamma}=\hbar \omega$ is the photon energy and $\theta_{\mathrm{t}}$ is the propagation angle with respect to the direction of the local magnetic field.

Recent discovery of high-magnetic-field pulsars (HBPs) however has challenged the existing pair creation theories. Few HBPs are seen to have the inferred surface dipolar fields above the photon splitting level: PSRs J1119-6127, J1814-1744 and J1726-3530 (Table 1). Yet another strong-field neutron star PSR J1846-0258 with $B_{\mathrm{d}} \sim 5 \times 10^{13} \mathrm{G}$ was discovered (Gotthelf et al. 2000), which seems to be radio-quiet (Kaspi et al. 1996), although its X-ray emission is apparently driven by dense $\mathrm{e}^{-} \mathrm{e}^{+}$pair plasma (e.g. Cordes 2001). Bearing in mind that the actual threshold due to photon splitting and/or bound positronium formation can be well below the critical field $B_{\mathrm{cr}} \sim 4 \times 10^{13} \mathrm{G}$, all high-magnetic-field radio pulsars with $B_{\mathrm{d}}>10^{13} \mathrm{G}$ pose a challenge. To evade the photon splitting problem for these pulsars Zhang \& Harding (Zhang \& Harding 2000a, ZH00 hereafter) proposed "a unified picture for HBPs and magnetars". They argued that radio-quiet magnetars cannot have active inner accelerators (thus no $\mathrm{e}^{-} \mathrm{e}^{+}$pair production), while the HBPs can, with a difference attributed to the relative orientations of rotation and magnetic axes (neutron stars can be either parallel rotators (PRs) with $\boldsymbol{\Omega} \boldsymbol{B}_{\mathrm{p}}>0$ or antiparallel rotators (APRs) with $\boldsymbol{\Omega} \boldsymbol{B}_{\mathrm{p}}<0$, where $\boldsymbol{\Omega}$ is the pulsar spin axis and $\boldsymbol{B}_{\mathrm{p}}$ is the magnetic field at the pole). If the photon splitting suppresses completely the pair production at the polar cap surface, 
Table 1. Radio-loud HBPs with inferred magnetic field $B_{\mathrm{p}}=$ $6.4 \times 10^{19}(P \dot{P})^{1 / 2} \mathrm{G}$ higher than the critical quantum field $B_{\text {cr }}=4.4 \times 10^{13} \mathrm{G}$ (after Table 1 in Zhang \& Harding 2000b).

\begin{tabular}{llll}
\hline \hline source & $P(\mathrm{~s})$ & $\dot{P}(\mathrm{~s} / \mathrm{s})$ & $B_{p}(\mathrm{G})$ \\
\hline PSR J1814-1744 & 3.98 & $7.43 \times 10^{-13}$ & $1.1 \times 10^{14}$ \\
PSR J1119-6127 & 0.41 & $4.02 \times 10^{-12}$ & $8.2 \times 10^{13}$ \\
PSR J1726-3530 & 1.11 & $1.22 \times 10^{-12}$ & $7.4 \times 10^{13}$ \\
\hline
\end{tabular}

then the VG inner accelerator cannot form, since the high potential drop cannot be screened at the top of the acceleration region. Hence, ZH00 argued that in the high magnetic field regime $\left(B_{\mathrm{d}}>B_{\mathrm{cr}}\right)$ the pair production process is possible only if the SCLF accelerator forms. In fact, such SCLF accelerators are typically quite long and their pair formation front (PFF) can occur at high altitudes $r$, where the dipolar magnetic field $B_{\mathrm{d}} \propto r^{-3}$ has degraded below $B_{\text {cr }}$. Furthermore, ZH00 demonstrated that such a lengthened SCLF accelerator in a magnetar environment can form only for PRs and not for APRs. Consequently they concluded that the radio-loud HBPs are PRs with developed lengthened SCLF accelerator, while the radio-quiet magnetars (AXPs and SGRs) are APRs. It is worth emphasizing here that ZH00 developed their model under the assumption that the magnetic field at the surface of HBPs is purely dipolar.

In this paper we propose an alternative model for radio-loud HBPs based on a highly non-dipolar surface magnetic field, in which the photon splitting within the VG inner acceleration region does not operate even if the dipole magnetic field exceeds the critical value $B_{\mathrm{cr}}$ at the polar cap. Thus our model requires that HBPs are APRs, which is a consequence of the VG scenario (e.g. Ruderman \& Sutherland 1975; Gil \& Mitra 2001). This model is a follow-up of Gil \& Mitra (2001), who argued that the VG can form if the actual surface magnetic field is about $10^{13}$ Gauss. In other words, they assumed that all VG-driven radio pulsars require strong highly nondipolar surface magnetic fields, with strength $B_{\mathrm{s}}$ being more or less independent of the value of the global dipole field inferred from the magnetic braking law. Thus, if $B_{\mathrm{d}} \ll 10^{13} \mathrm{G}$ then $B_{\mathrm{s}} \gg B_{\mathrm{d}}$ and if $B_{\mathrm{d}} \gtrsim 10^{13} \mathrm{G}$ then $B_{\mathrm{s}} \sim B_{\mathrm{d}}$. However, for radio pulsars to operate, in any case $B_{\mathrm{s}}<B_{\mathrm{cr}} \sim B_{\mathrm{q}}$. We argue that such strong surface field anomalies can increase the dipolar field $B_{\mathrm{d}} \ll B_{\mathrm{cr}}$ in normal pulsars to values exceeding $10^{13} \mathrm{G}$ (required by the conditions for VG formation - see Gil \& Mitra 2001) if the global and local surface fields have the same polarities, or reduce the very high dipolar field $B_{\mathrm{d}} \gtrsim B_{\mathrm{cr}}$ in HBPs, if both these components are of comparable values and have opposite polarities.

\section{Modelling the surface magnetic field}

We model the actual surface magnetic field by superposition of the star-centered global dipole $\boldsymbol{d}$ and a crustanchored dipole moment $\boldsymbol{m}$, whose influence results in

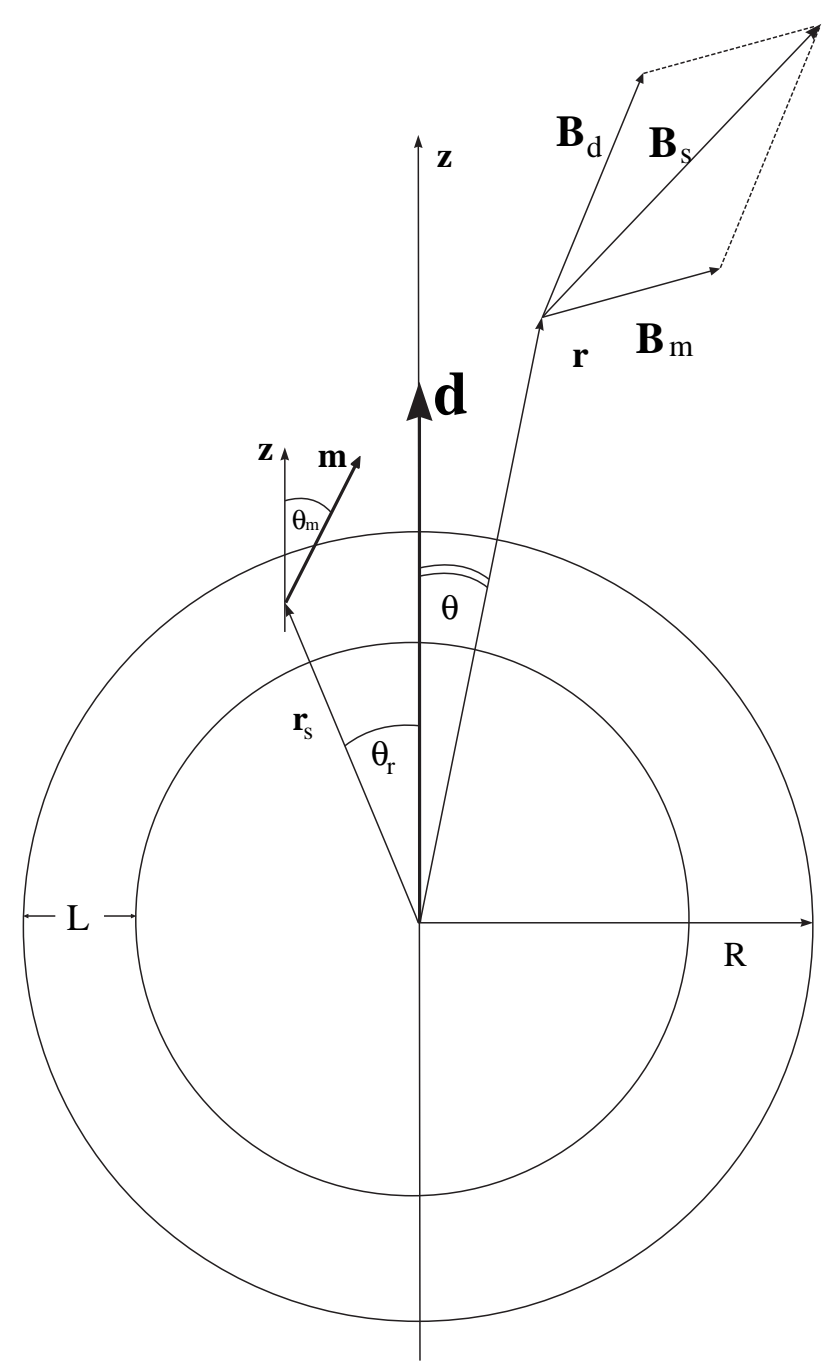

Fig. 1. Superposition of the star-centered global magnetic dipole "d" and crust-anchored local dipole " $m$ " placed at $\boldsymbol{r}_{\mathrm{s}}=\left(r_{\mathrm{s}} \sim R, \theta=\theta_{\mathrm{r}}\right)$ and inclined to the $z$-axis by an angle $\theta_{\mathrm{m}}$. The actual surface magnetic field at radius vector $\boldsymbol{r}=(r, \theta)$ is $\boldsymbol{B}_{\mathrm{s}}=\boldsymbol{B}_{\mathrm{d}}+\boldsymbol{B}_{\mathrm{m}}$, where $B_{\mathrm{d}}=2 d / r^{3}, B_{\mathrm{m}}=2 m /\left|\boldsymbol{r}-\boldsymbol{r}_{\mathrm{s}}\right|^{3}$, $r$ is the radius (altitude) and $\theta$ is the polar angle (magnetic colatitude). $R$ is the radius of the neutron star and $L$ is the crust thickness.

small-scale deviations of the surface magnetic field from the global dipole, as presented in Fig. 1. The technical details of the calculations of the resultant surface magnetic field are presented in the Appendix. Here we discuss the main results and their consequences. For simplicity, in this paper we mostly consider an axially symmetric case in which both $\boldsymbol{d}$ and $\boldsymbol{m}$ are directed along the $z$-axis (parallel or antiparallel), thus $\theta_{\mathrm{r}}=\theta_{\mathrm{m}}=\phi_{\mathrm{r}}=\phi_{\mathrm{m}}=0$ (see Fig. 1). Also, for convenience " $\boldsymbol{m}$ " is expressed in units of $\boldsymbol{d}$. We use normalized units in which $d=P=R=1$ and $r_{\mathrm{s}}=0.95$. (see caption of Fig. 2 for the normalization convention). All calculations are carried out in threedimensions, although, for clarity of graphic presentation, in the figures we present only two-dimensional cuts of the open field line regions. 


\subsection{High-magnetic-field pulsars}

As mentioned above, the formation of the VG inner accelerator requires a very high magnetic field $B_{\mathrm{S}} \gtrsim 10^{13} \mathrm{G}$ on the surface of the polar cap (Usov \& Melrose 1995, 1996; Gil \& Mitra 2001). This can be achieved not only in pulsars with high dipolar field $B_{\mathrm{d}} \gtrsim 10^{13} \mathrm{G}$. In fact, some of the low-field pulsars with $B_{\mathrm{d}} \ll 10^{13} \mathrm{G}$ can have a surface field $B_{\mathrm{s}} \gtrsim 10^{13} \mathrm{G}$ if $B_{\mathrm{m}} \gg B_{\mathrm{d}}$ (thus $m \gg 1.25 \times 10^{-4} \mathrm{~d}$ ). We discuss such normal, lowfield pulsars later in this paper. Presently let us consider the HBP with a dipolar surface field at the pole $B_{\mathrm{d}}=6.4 \times 10^{19}(P \cdot \dot{P})^{1 / 2} \mathrm{G}$ exceeding the photon splitting limit $B_{\mathrm{cr}} \sim B_{\mathrm{q}}$. If all photon splitting modes operate, such a pulsar should be radio-quiet. Alternatively these pulsars could be radio-loud if the effective surface field is reduced below $B_{\mathrm{cr}}$. Such a scenario can be achieved if the polarities of magnetic moments $\boldsymbol{d}$ and $\boldsymbol{m}$ are opposite, that is $\boldsymbol{d}$ and $\boldsymbol{m}$ are antiparallel. Figure 2 presents a case with $\boldsymbol{m}=-10^{-4} \boldsymbol{d}$ and $\Delta R / R=0.05$. The actual surface values of $B_{\mathrm{s}}=\sqrt{\left(B_{\mathrm{r}}^{\mathrm{s}}\right)^{2}+\left(B_{\theta}^{\mathrm{s}}\right)^{2}}$ as well as radial components of $B_{\mathrm{r}}^{\mathrm{s}}=\boldsymbol{B}_{\mathrm{s}} \cdot \boldsymbol{R} / R$ and $B_{\mathrm{r}}^{\mathrm{d}}=\boldsymbol{B}_{\mathrm{d}} \cdot \boldsymbol{R} / R$ are presented in the lower panel of Fig. 2 (note that all radial components are positive and that the total $B_{\mathrm{s}}$ is almost equal to $B_{\mathrm{r}}^{\mathrm{s}}$ in this case). At the pole (radius $r=R$ and polar angle $\theta=0)$ the ratio $B_{\mathrm{m}} / B_{\mathrm{p}}=(\mathrm{m} / d) \cdot(R / \Delta R)^{3}=0.8$ and thus $B_{\mathrm{s}}=B_{\mathrm{p}}-B_{\mathrm{m}}=B_{\mathrm{d}}(1-0.8)=0.2 B_{\mathrm{p}}$. As one can see from this figure, all surface field lines between $-\theta_{\mathrm{s}}$ and $+\theta_{\mathrm{s}}$ are open, but the ratio $B_{\mathrm{s}} / B_{\mathrm{d}}$ increases towards the polar cap edge, reaching a value of about 0.5 in the region between polar angles $\left|\theta_{\mathrm{d}}\right|$ and $\left|\theta_{\mathrm{s}}\right|$. The ratio $B_{\mathrm{m}} / B_{\mathrm{d}}$ is also about 0.5 in this region. Thus, the global dipolar field $\left(B_{\mathrm{d}}=2\right.$ in our units) is effectively reduced by between 2 and 5 times in different parts of the polar cap (defined as the surface area from which the open magnetic field lines emanate). This means that the ratio $B_{\mathrm{m}} / B_{\mathrm{d}}$ ranges from 0.5 to 0.8 across the polar cap. The actual polar cap is broader than the canonical dipolar polar cap (two dashed vertical lines correspond to the last open dipolar field lines emanating at the polar angles $\theta_{\mathrm{d}}= \pm 0.014$ radians for a typical period $P=1 \mathrm{~s}$ ). The ratio of actual to dipolar polar cap radii is $\theta_{\mathrm{s}} / \theta_{\mathrm{d}} \sim 5 / 3$ in this case. Thus, using the argument of magnetic flux conservation of the open field lines, one can say that an effective surface magnetic field of the polar cap is about 2.8 times lower than the dipolar surface field measured from the values of $P$ and $\dot{P}$. If the estimated dipole field $B_{\mathrm{p}} \approx 10^{14} \mathrm{G}$ (like in the case of PSR J1814-1744, Table 1) then the actual surface field at the pole is only $B_{\mathrm{s}} \sim 2.5 \times 10^{13} \mathrm{G}$, well below the photon splitting death line $B_{\mathrm{cr}}=4.4 \times 10^{13} \mathrm{G}$. Such a pulsar can be radio-loud without invoking the lengthened SCLF accelerator proposed by ZH00. As shown by Gil \& Mitra (2001), in such a strong surface magnetic field the vacuum gap accelerator can form, which implies low altitude coherent radio emission (Melikidze et al. 2000, see Sect. 3 in this paper) at altitudes $r_{\mathrm{em}} \sim 50 R$ (for a typical pulsar with $P=1 \mathrm{~s}$ ) in agreement with observational constraints

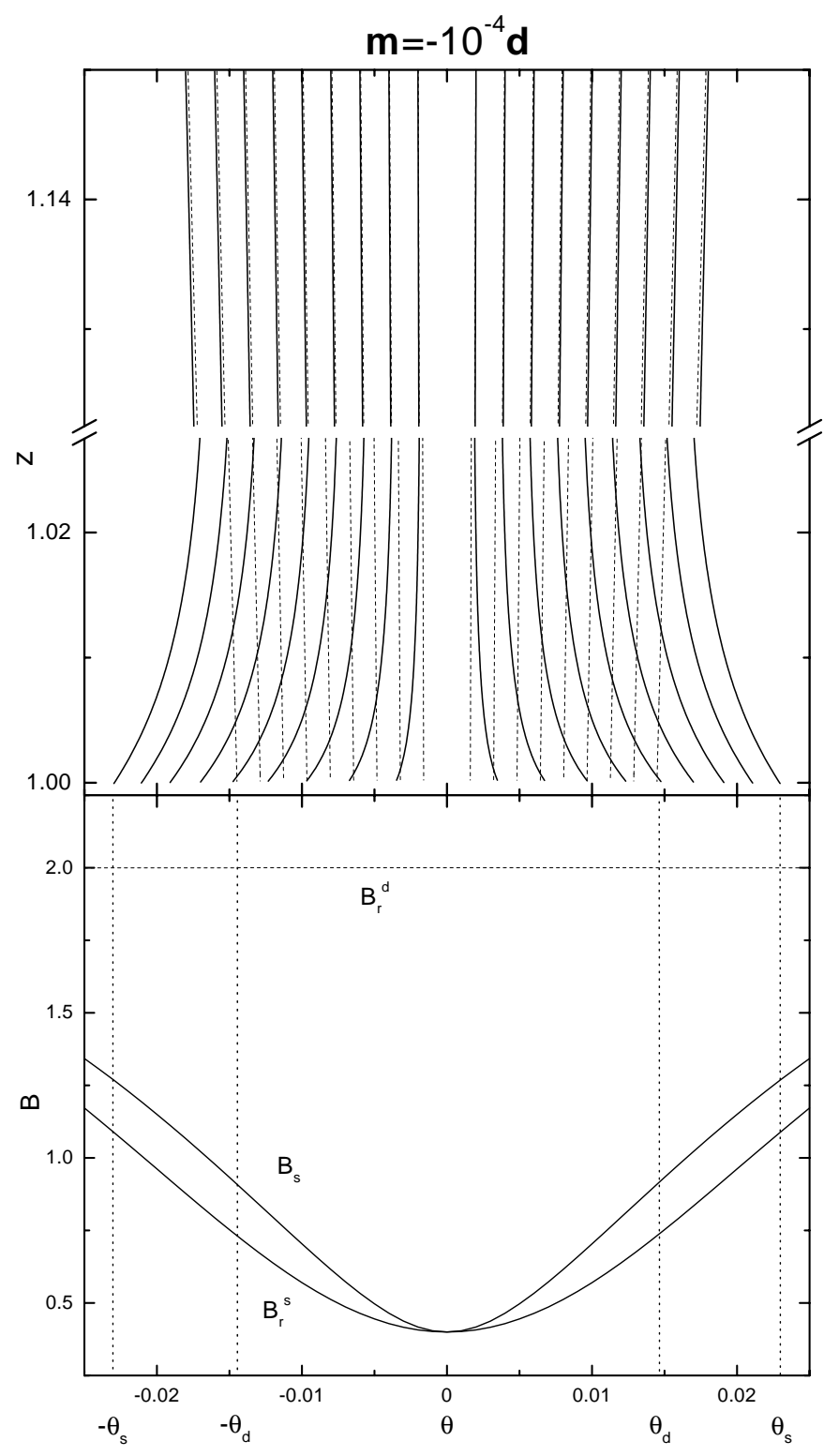

Fig. 2. Structure of the surface magnetic field for a superposition of the global star-centered dipolar moment $\boldsymbol{d}$ and crustanchored dipole moment $\boldsymbol{m}=-10^{-4} \boldsymbol{d}$ (Fig. 1). The open dipolar field lines (solid) and the actual surface open field lines (dashed) are shown in the upper panel. The horizontal axis is labelled as azimuthal angle $\theta$ (magnetic colatitude), which measures the polar cap radius. For purely dipolar field lines the polar cap radius $r_{\mathrm{d}} \approx R \cdot \sin \theta_{\mathrm{d}}$, which for pulsar period $P=1 \mathrm{~s}$ is about 0.014 radians (thus $r_{\mathrm{d}} \approx 1.4 \times 10^{4} \mathrm{~cm}$ ). The actual polar cap is broader, with the last open lines emanating at the polar angles $\theta_{\mathrm{s}} \approx 0.023$ (thus the actual polar cap radius $r_{\mathrm{s}}=2.3 \times 10^{4} \mathrm{~cm}=1.65 r_{\mathrm{d}}$ ). The actual open surface field lines (solid) reconnect with dipolar field lines (dashed) at distances $z=(r / R) \cdot \cos \theta \approx 1.2$, where $r$ is the radius and $\theta<0.025$ radians. In the lower panel the surface values $(r=R)$ of both dipolar field (dashed horizontal) and the actual field (solid line) are shown. The radial components $B_{\mathrm{r}}^{\mathrm{d}}=2 d \cos \theta / R^{3} \approx 2$ and $B_{\mathrm{r}}^{\mathrm{s}}=\left(\boldsymbol{B}_{\mathrm{d}}+\boldsymbol{B}_{\mathrm{m}}\right) \cdot \boldsymbol{r} / r$, and total values $B_{\mathrm{s}}=\sqrt{\left(B_{\mathrm{r}}^{\mathrm{s}}\right)^{2}+\left(B_{\theta}^{\mathrm{s}}\right)^{2}}$ are presented (where $d=R=1$ is assumed for convenience). 


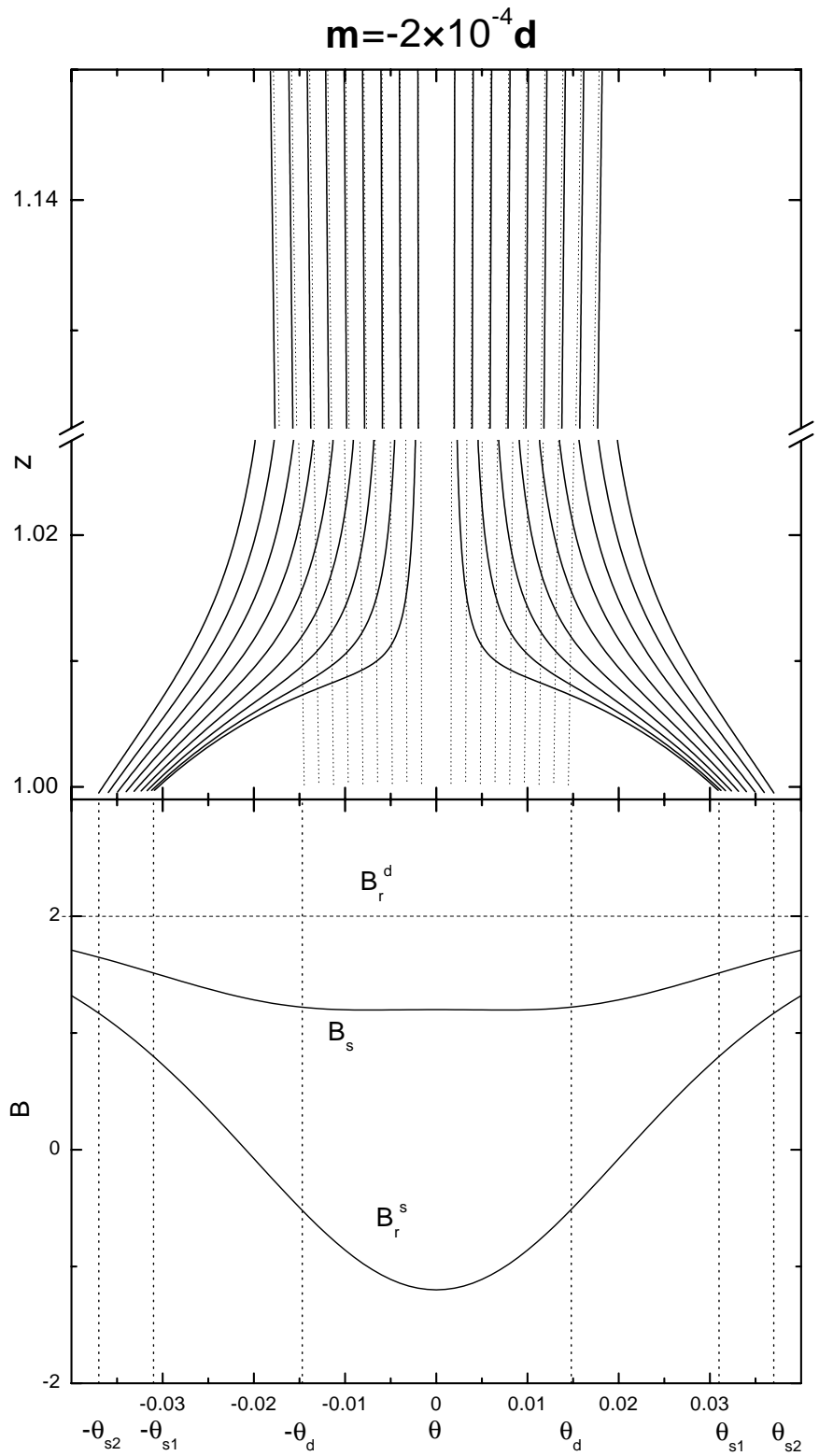

Fig. 3. As in Fig. 2 but for $\boldsymbol{m}=-2 \times 10^{-4} \boldsymbol{d}$.

on radio emission altitudes (Cordes 1978, 1992; Kijak \& Gil 1997, 1998; Kijak 2001).

Figure 3 presents another case of opposite polarities $\boldsymbol{m}=-2 \times 10^{-4} \boldsymbol{d}$, with magnitude of $\boldsymbol{m}$ being two times stronger than in the previous case (Fig. 2). Again for $\Delta R / R \sim 0.05, B_{\mathrm{m}} / B_{\mathrm{p}}=(m / d)(R / \Delta R)^{3}=1.6$ at the pole $(r=R$ and $\theta=0)$ and $B_{\mathrm{s}}=B_{\mathrm{p}}-B_{\mathrm{m}}=-0.6 B_{\mathrm{p}}$. The negative sign of the ratio $B_{\mathrm{s}} / B_{\mathrm{d}}$ means that the surface magnetic field $\boldsymbol{B}_{\mathrm{s}}$ is directed opposite to $\boldsymbol{B}_{\mathrm{d}}$ near the pole, that is the circumpolar field lines with polar angles $-\theta_{\mathrm{s} 1}<$ $\theta<\theta_{\mathrm{s} 1}$ (where $\theta_{\mathrm{s} 1} \sim 0.031$ ) are closed. The last open surface field lines (solid) emanating at polar angles $\theta_{\mathrm{s}}= \pm \theta_{\mathrm{s} 2}$ (where $\theta_{\mathrm{s} 2} \sim 0.037$ ) reconnect with the last open dipolar field lines (dashed) at altitudes $z \sim 1.2$ (thus about $2 \mathrm{~km}$ above the surface). The actual polar cap, which is the surface through which the open magnetic field lines emanate, has the shape of a ring $\left(0.031 \lesssim\left|\theta_{\mathrm{S}}\right| \lesssim 0.037\right)$ located outside the circle of the dipolar polar cap with angular radius $\theta_{\mathrm{d}}=0.014$ (or diameter $r_{\mathrm{d}} \approx \theta_{\mathrm{d}} \cdot R \approx 1.4 \times 10^{4} \mathrm{~cm}$ ). Again, the magnetic flux conservation argument leads to $B_{\mathrm{s}} / B_{\mathrm{d}}=\left(\theta_{\mathrm{s}_{2}}^{2}-\theta_{\mathrm{s}_{1}}^{2}\right) / \theta_{\mathrm{d}}^{2}=\left(0.037^{2}-0.031^{2}\right) / 0.014^{2}=0.48$, thus $B_{\mathrm{s}}$ is about $0.5 B_{\mathrm{d}}$ within the ring of the open field lines (note that $\left|B_{\mathrm{m}}\right| / B_{\mathrm{d}} \sim 1.6$ in this region). The actual values of the surface magnetic field (radial $B_{\mathrm{r}}^{\mathrm{s}}$ and total $\left.B_{\mathrm{s}}=\sqrt{\left(B_{\mathrm{r}}^{\mathrm{s}}\right)^{2}+\left(B_{\theta}^{\mathrm{s}}\right)^{2}}\right)$ are shown as solid lines in the lower panel of Fig. 3, in comparison with radial components of the dipolar field $B_{\mathrm{d}}$ (dashed horizontal line). As one can see, $B_{\mathrm{r}}^{\mathrm{s}}<B_{\mathrm{r}}^{\mathrm{d}}=2 m \cos \theta / R^{3}$ and $B_{\mathrm{r}}^{\mathrm{s}}$ is negative for $|\theta|<0.022$. If the dipolar surface component of the pulsar magnetic field is $B_{\mathrm{d}} \sim 7 \times 10^{13} \mathrm{G}$ (like PSR J1726-3530 given in Table 1), then the actual surface magnetic field $B_{\mathrm{S}} \sim 4 \times 10^{13} \mathrm{G}$, is below the photon splitting threshold.

\subsection{Normal pulsars}

Figure 4 presents a case with $\boldsymbol{m}=2 \times 10^{-4} \boldsymbol{d}$ in which both magnetic moments have the same polarity. Obviously, in such a case the surface magnetic field will be stronger as compared with pure dipole $(m=0)$. The flux conservation argument gives a surface magnetic field $B_{\mathrm{s}} / B_{\mathrm{d}}=$ $\left(\theta_{\mathrm{d}} / \theta_{\mathrm{s}}\right)^{2}=(0.014 / 0.008)^{2} \sim 3$ (the ratio $\left.B_{\mathrm{m}} / B_{\mathrm{d}} \lesssim 1.6\right)$. Thus the actual surface field is about 3 times stronger than the inferred dipolar field $B_{\mathrm{p}}=6.4 \times 10^{19}(P \cdot \dot{P})^{1 / 2} \mathrm{G}$. Such cases of increasing an effective magnetic field can be important in normal pulsars with low dipolar field $B_{\mathrm{d}} \ll 10^{13} \mathrm{G}$ (Gil \& Mitra 2001).

It is then interesting to examine how different polarities of $\boldsymbol{d}$ and $\boldsymbol{m}$ would influence normal pulsars with $B_{\mathrm{d}} \ll B_{\text {cr }}$. If $m / d \sim(\Delta R / R)^{3}$ thus $B_{\mathrm{m}} \sim B_{\mathrm{d}}$ then of course $B_{\mathrm{s}}$ can be slightly lower than $B_{\mathrm{d}}$, as in the case of HBPs (Fig. 2). In such a case, however, the VG cannot form. In fact, as argued by Gil \& Mitra (2001), the formation of the VG requires that $B_{\mathrm{s}}$ is close to $10^{13} \mathrm{G}$ or even above, thus $B_{\mathrm{m}} \gg B_{\mathrm{d}}$ is required in normal pulsars (see also Gil et al. 2001). Figure 5 illustrates a case of high surface magnetic field with $B_{\mathrm{m}}=3.2 B_{\mathrm{p}}$, in which the VG can apparently form. As one can see from this figure, the values of $B_{\mathrm{s}}$ at the ring-shaped polar cap are close to dipolar values $B_{\mathrm{s}}=B_{\mathrm{d}}(r=R,|\theta| \sim 0.05)$. One can show that this is a general situation, that is, $B_{\mathrm{s}} \sim B_{\mathrm{d}}$ no matter by how much $B_{\mathrm{m}}$ exceeds $B_{\mathrm{d}}$ at the pole. This follows from the fact that the angular location $\theta$ of the polar cap ring increases with increasing ratio $B_{\mathrm{m}} / B_{\mathrm{d}} \approx(m / d)(\Delta R / R)^{3} \simeq 8 \times 10^{3}(\mathrm{~m} / d)$. For example, in the case presented in Fig. $5 \boldsymbol{m}=-4 \times 10^{-4} \boldsymbol{d}$ and the last open field lines emanate at polar angles $\theta_{\mathrm{s}} \approx \pm 0.055$ radians, or at polar cap radii $R_{\mathrm{p}} \sim 6 \times 10^{4} \mathrm{~cm}$ (for $P=1 \mathrm{~s}$ ). Thus, the narrow polar cap ring is located far from the local dipole $\boldsymbol{m}$, whose influence is weak at this distance. The circumpolar field lines between polar angles -0.053 to +0.053 are closed.

Thus, we conclude that the actual pulsar surface magnetic field $B_{\mathrm{s}}$ can significantly differ (say by an order of magnitude) from the inferred dipolar field $B_{\mathrm{d}}$ only in the 


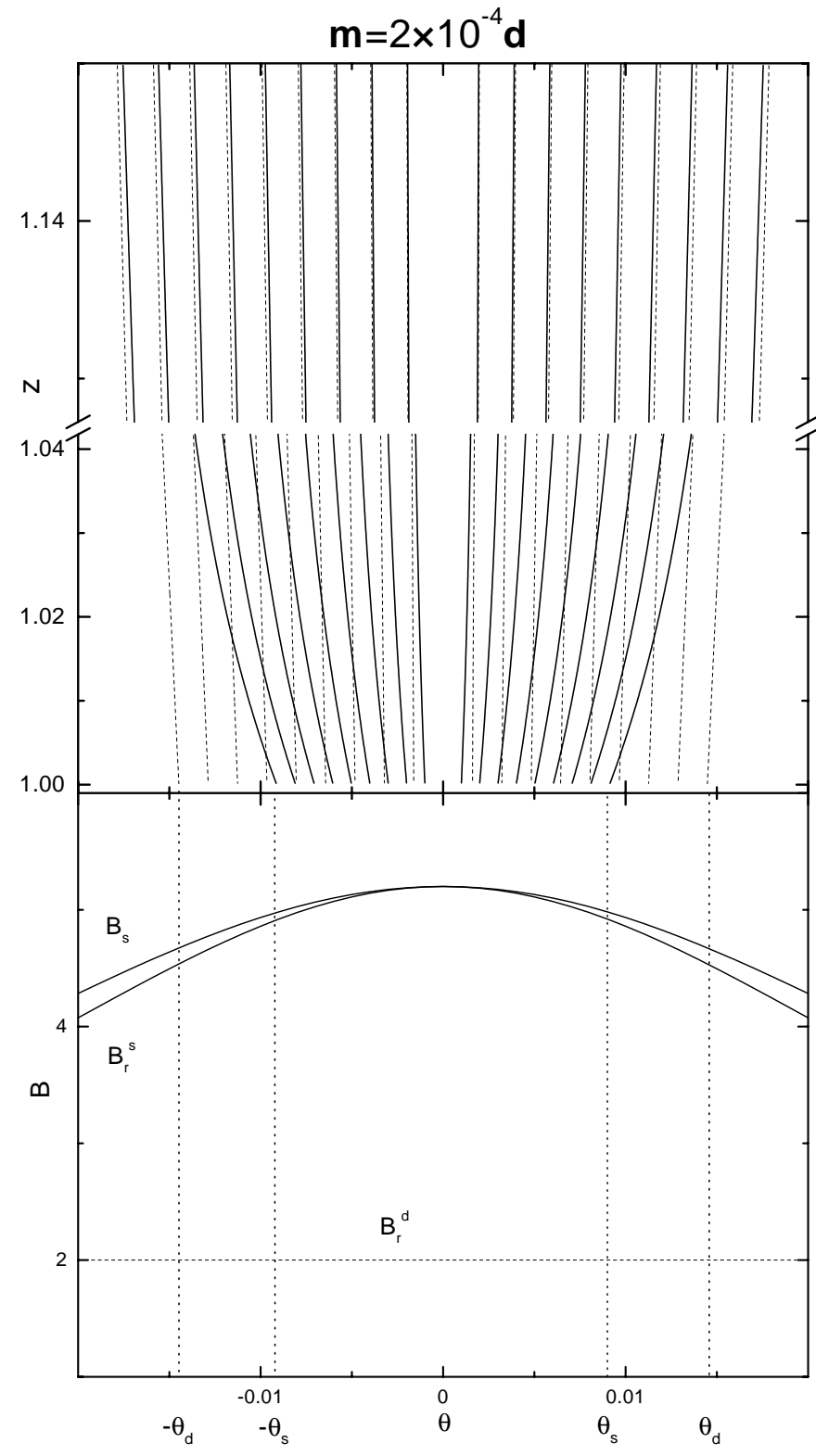

Fig. 4. As in Fig. 3 but for $\boldsymbol{m}=2 \times 10^{-4} \boldsymbol{d}$.

case when the polarities of the global $\boldsymbol{d}$ and local $\boldsymbol{m}$ dipole (Fig. 1) are the same, as illustrated in Fig. 4. If this is the case, then $B_{\mathrm{s}}$ can largely exceed $B_{\mathrm{d}}$, which seems to be important from the viewpoint of vacuum gap formation, which requires $B_{\mathrm{s}} \gtrsim 10^{13} \mathrm{G}$ (see Gil \& Mitra 2001). Therefore, in normal VG-driven radio pulsars the polar cap should be circular, or at least filled - if the axial symmetry does not hold. A ring-shaped polar cap can occur only in normal pulsars with $B_{\mathrm{d}} \lesssim B_{\text {cr }}$ and in radio-loud HBPs with $B_{\mathrm{d}} \gtrsim B_{\mathrm{cr}}$.

In the accompanying paper Gil et al. (2002) explored consequences of the vacuum gap model interpretation for drifting subpulses observed in PSR B0943+10, in which 20 sparks move circumferentially around the perimeter of the polar cap, each completing one circulation in 37 pulsar periods (Deshpande \& Rankin 1999, 2001). Gil et al. (2002) considered both the curvature radiation (CR)

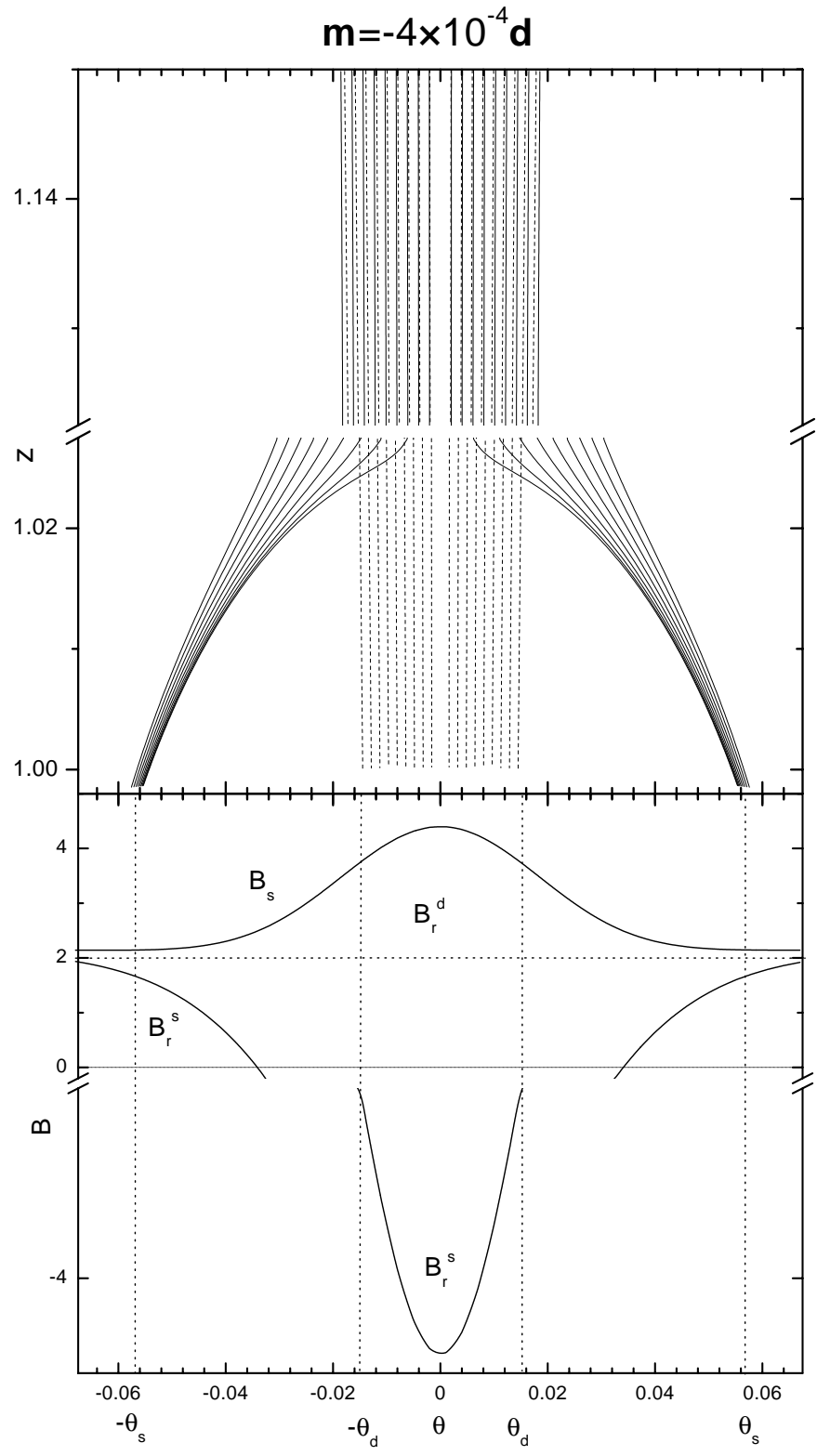

Fig. 5. As in Fig. 4 but for $\boldsymbol{m}=-4 \times 10^{-4} \boldsymbol{d}$.

and resonant inverse Compton radiation (ICS) seed photons as sources of electron-positron pairs and determined the parameter space for the surface magnetic field structure in each case. For the CR-VG the surface magnetic field strength $B_{\mathrm{s}}>2 \times 10^{13} \mathrm{G}$ and the radius of curvature of surface field lines $0.6 \times 10^{5} \mathrm{~cm}<\mathcal{R}<1.2 \times 10^{5} \mathrm{~cm}$, while for the resonant ICS-VG $B_{\mathrm{s}}>2 \times 10^{13} \mathrm{G}$ and $10^{6} \mathrm{~cm}<\mathcal{R}<3 \times 10^{6} \mathrm{~cm}$ (of course, in both caseS $B_{\mathrm{s}}<B_{\mathrm{q}} \sim 4.4 \times 10^{13} \mathrm{G}$ ). The CR-VG with such a curved surface magnetic field does not seem likely (although it cannot be excluded), while the ICS-VG gap supported by the magnetic field structure determined by the parameter space specified above guarantees a system of 20 sparks circulating around the perimeter of the polar cap by means of the $\boldsymbol{E} \times \boldsymbol{B}$ drift in about 37 pulsar periods.

Further, Gil et al. (2002) modelled the magnetic field structure determined by the ICS-VG parameter space 
(specified above), using the numerical formalism developed in this paper. Since $B_{\mathrm{p}}=6.4 \times 10^{19}(P \cdot \dot{P})^{1 / 2} \mathrm{G}=$ $4 \times 10^{12} \mathrm{G}$ in this case, to obtain $\boldsymbol{B}_{\mathrm{s}} \sim(2 \div 3) \times 10^{13} \mathrm{G}$ one needs $B_{\mathrm{m}} \gg B_{\mathrm{d}}$ and the same polarity of both components. Following the symmetry suggested by the observed patterns of drifting subpulses in PSR B0943+10, the local dipole axis was placed at the polar cap center. A number of model solutions corresponding to $r_{\mathrm{s}} \sim 0.97$ and $m \sim(1 \div 2) \times 10^{-4} \mathrm{~d}$ and satisfying the ICS-VG parameter space, was then obtained. As a result of this specific modelling Gil et al. (2002) obtained a number of interesting and important conclusions: (i) The conditions for the formation of the ICS-VG are satisfied only at the peripheral ring-like region of the polar cap, which can just accommodate a system of 20 sparks performing $\boldsymbol{E} \times \boldsymbol{B}$ drift. (ii) The surface magnetic field lines within the actual gap are converging, which stabilizes the $\boldsymbol{E} \times \boldsymbol{B}$ drifting sparks by preventing them from rushing towards the pole, as opposed to the case of a diverging dipolar field (e.g. Fillipenko \& Radhakrishnan 1982). (iii) No model solutions with $B_{\mathrm{s}} \sim(3 \div 4) \times 10^{13} \mathrm{G}$ and $\mathcal{R} \sim(0.6 \div 1.2) \times 10^{5} \mathrm{~cm}$, could be obtained which corresponding to the CR-VG parameter space, which in turn favors the ICS-VG in PSR B0943+10.

\section{Discussion and conclusions}

We argue in this paper that the presence of a strong non-dipolar magnetic field on the neutron star surface can help to understand the recently discovered radio pulsars with dipolar magnetic field above the photon splitting threshold, as well as to understand the long-standing problems of vacuum gap formation and drifting subpulse phenomenon. We model the actual surface magnetic field as a superposition of the global star-centered (large-scale) dipole and local crust-anchored (small-scale) dipoles $\boldsymbol{B}_{\mathrm{s}}=$ $\boldsymbol{B}_{\mathrm{d}}+\sum_{i} \boldsymbol{B}_{\mathrm{m} i} \approx \boldsymbol{B}_{\mathrm{d}}+\boldsymbol{B}_{\mathrm{MO}}$, where $\boldsymbol{B}_{\mathrm{MO}}$ is the local dipole nearest to the polar cap centre (Fig. 1). Such a model is quite general, as it describes the magnetic field structure even if the star-centered fossil dipole field is negliglible at the star surface. In such a case the global surface dipole field $\boldsymbol{B}_{\mathrm{d}}$ (inferred from $P$ and $\dot{P}$ measurements) is a superposition of all crust-anchored dipoles calculated at a far distance and projected down to the polar cap surface according to the dipolar law.

We propose a model for radio-loud HBPs with high inferred dipolar magnetic field $B_{\mathrm{d}}>10^{13} \mathrm{G}$, even exceeding the critical value $B_{\text {cr }} \sim 4 \times 10^{13}$ G. Given the difficulty that in a strong magnetic field the magnetic pair creation process is largely suppressed, the puzzling issue remains how these HBPs produce their $\mathrm{e}^{-} \mathrm{e}^{+}$pair plasma necessary for the generation of the observable radio emission. Zhang \& Harding (2000a) proposed a "lengthened version" of the stationary SCLF model of inner accelerator (e.g. Arons \& Sharleman 1979), in which the pair formation front occurs at altitudes $r$ high enough above the polar cap that $B_{\mathrm{d}} \sim B_{\mathrm{cr}}(R / r)^{3}$ degrades below $B_{\mathrm{cr}}$, thus evading the photon splitting threshold. Our VG model is an alternative to the lengthened SCLF model, with pair creation occurring right at the polar cap surface, even if the magnetic field exceeds $B_{\mathrm{cr}}$. We have assumed that the open surface magnetic field lines result in an actual pulsar from superposition of the star-centered global dipole moment and a crust-anchored local dipole moment. We argued that if the polarities of these two components are opposite, and their values are comparable, then the actual value of the surface magnetic field $B_{\mathrm{s}}$ can be lower than the critical field $B_{\mathrm{cr}}$, even if the global dipole field $B_{\mathrm{d}}$ exceeds the critical value. Thus, the creation of electron-positron plasma is possible at least over a part of the polar cap and these high magnetic field neutron stars can be radioloud (HBPs). In fact, one should expect that in HBPs, in which by definition $B_{\mathrm{d}} \gtrsim B_{\mathrm{cr}} \sim B_{\mathrm{q}}=4.4 \times 10^{13} \mathrm{G}$, the ratio $B_{\mathrm{m}} / B_{\mathrm{d}}$ should be of the order of unity, since $\boldsymbol{B}_{\mathrm{s}}=\boldsymbol{B}_{\mathrm{d}}+\boldsymbol{B}_{\mathrm{m}}$ and $10^{13} \mathrm{G} \lesssim B_{\mathrm{s}} \lesssim B_{\mathrm{cr}} \sim 4 \times 10^{13} \mathrm{G}$.

Within our simple model of a non-dipolar surface magnetic field $\boldsymbol{B}_{\mathrm{s}}$ one should expect that both cases $\boldsymbol{m} \cdot \boldsymbol{d}>0$ and $\boldsymbol{m} \cdot \boldsymbol{d}<0$ will occur with approximately equal probability. However, from the viewpoint of observable radio emission only the latter case is interesting in HBPs with $B_{\mathrm{d}} \gtrsim B_{\mathrm{cr}}$. In fact, when $\boldsymbol{m} \cdot \boldsymbol{d}>0$, then the surface magnetic field $B_{\mathrm{s}}>B_{\mathrm{d}} \gg B_{\mathrm{cr}}$ (Fig. 4) and the photon splitting level is highly exceeded. For $\boldsymbol{m} \cdot \boldsymbol{d}<0$ we have two possibilities: (i) if $m / d \lesssim(\Delta R / R)^{3}$ thus $B_{\mathrm{m}} \lesssim B_{\mathrm{d}}$ at the pole $(r=R, \theta=0)$ then the polar cap (locus of the open field lines) is circular (Fig. 2); (ii) if $m / d>(\Delta R / R)^{3}$ thus $B_{\mathrm{m}}>B_{\mathrm{d}}$ then part of the circumpolar field lines are closed and the actual polar cap has the shape of ring (Fig. 3). In both cases (i) and (ii), the actual surface magnetic field $B_{\mathrm{s}}$ at the polar cap (or at least part of it) can be lower than $B_{\mathrm{cr}}$, even if $B_{\mathrm{d}}$ exceeds $B_{\mathrm{cr}}$. The values of $B_{\mathrm{m}}$ and $B_{\mathrm{d}} \gtrsim B_{\mathrm{cr}}$ should be comparable to make reduction of The strong surface field $B_{\mathrm{s}}$ below $B_{\mathrm{cr}}$ possible. In our illustrative examples presented in Figs. 2 and 3 (corresponding to the same pulsar with $P=1 \mathrm{~s}$ and $\left.B_{\mathrm{p}}=2 \mathrm{~d} R^{-3}=6.4 \times 10^{19}(P \cdot \dot{P})^{1 / 2} \mathrm{G}\right)$ we used ratios $B_{\mathrm{m}} / B_{\mathrm{d}}$ ranging from 0.5 to 1.6 . These values could be slightly different, say by a factor of a few, thus we can say that the ratio $B_{\mathrm{m}} / B_{\mathrm{d}}$ should be of the order of unity. If $B_{\mathrm{m}} / B_{\mathrm{d}} \gg 1$, then the reduction of the surface dipole field is not effective (see example presented and discussed in Fig. 5). On the other hand, the case with $B_{\mathrm{m}} \ll B_{\mathrm{d}}$ is not interesting, as it represents a weak surface magnetic field anomaly. Thus, among a putative population of neutron stars with $B_{\mathrm{d}} \gtrsim B_{\mathrm{cr}}$, only those with a ratio $B_{\mathrm{m}} / B_{\mathrm{d}}=(m / d)(R / \Delta R)^{3}$ of the order of unity, and with magnetic moment $\boldsymbol{m}$ and $\boldsymbol{d}$ (Fig. 1) antiparallel at the polar cap surface, that is $\boldsymbol{m} \cdot \boldsymbol{d}<0$, can be detected as HBPs. Other neutron stars from this population of high magnetic dipole field objects should be radio-quiet. This probably explains why there are so few HBPs detected.

Within the lengthened SCLF model there is an upper limit around $B_{\mathrm{d}}=2 \times 10^{14} \mathrm{G}$ for radio-loud HBPs (ZH00, ZH01, Zhang 2001). As ZH00 argued, detecting a pulsar above this limit would strongly imply that only one mode of photon splitting occurs. Without the alternative model of HBPs proposed in this paper, such a detection 


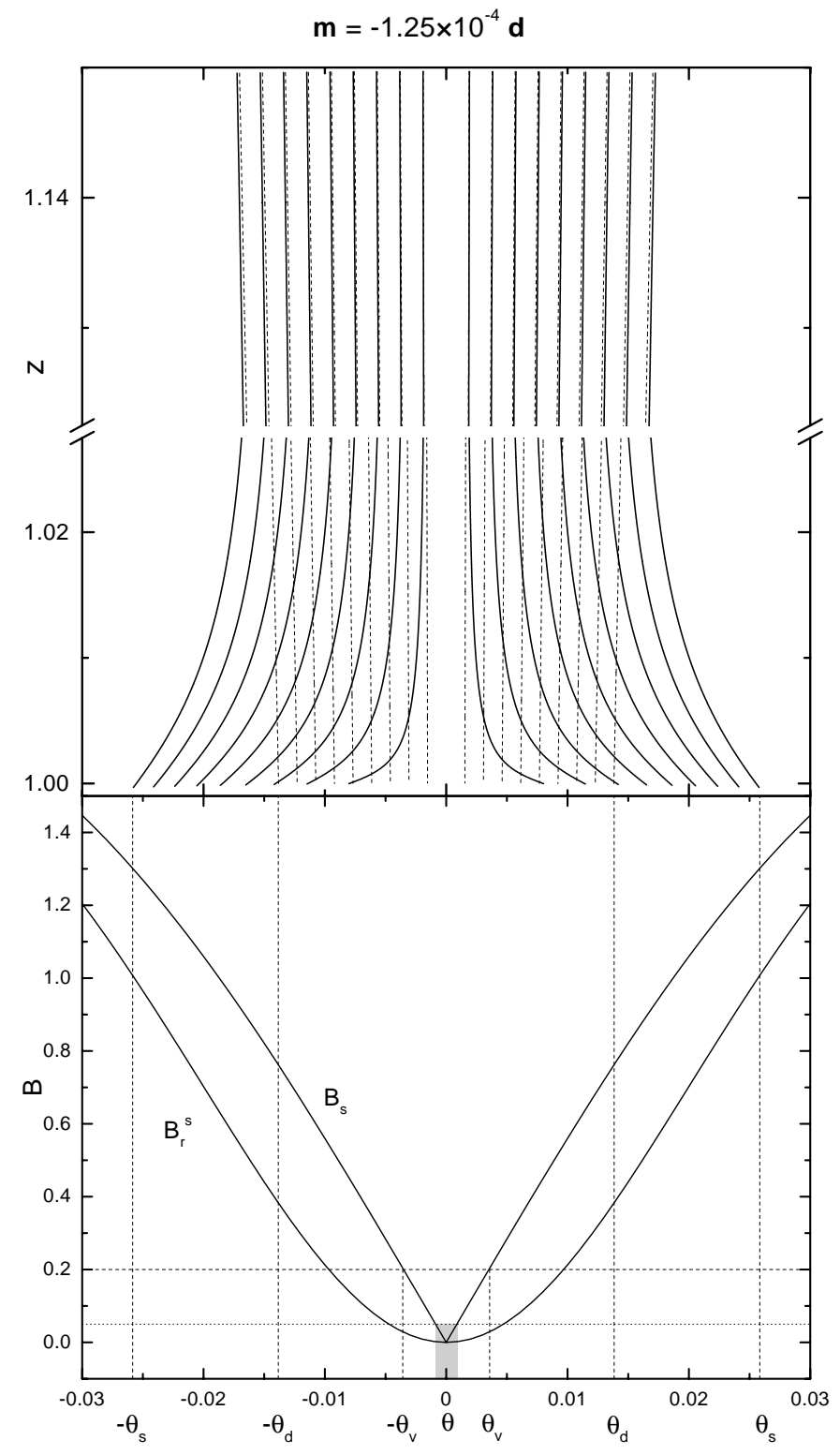

Fig. 6. As in Fig. 2 but for $\boldsymbol{m}=-1.25 \times 10^{-4} \boldsymbol{d}$. See also text for explanation.

would really be of great importance for the fundamental physics of the photon splitting phenomenon. In our VGbased model there is no natural upper limit for the radioloud HBPs. However, it is known that due to the magnetic pressure the neutron star surface would tend to "crack", which should occur at magnetic field strengths approaching $10^{15} \mathrm{G}$ (Thompson \& Duncan 1995). It is unclear how the radio emission would be affected by such a cracking process.

To illustrate the above argument, let us consider Fig. 6 which presents yet another case of opposite polarities $\boldsymbol{m}=-1.25 \times 10^{-4} \boldsymbol{d}$. With $\Delta R / R \sim 0.05$ this gives $B_{\mathrm{m}} / B_{\mathrm{p}}=1.0$ and $B_{\mathrm{s}}=B_{\mathrm{p}}-B_{\mathrm{m}}=0$ at the pole $(r=R, \theta=0)$. The dashed horizontal line at $B=0.2$ in the lower panel corresponds to the surface magnetic field $B_{\mathrm{s}}$ which is 10 times weaker than the global dipole component $B_{\mathrm{d}}=2$ (not shown in the figure). Thus if, for example, $B_{\mathrm{p}}=4 \times 10^{14} \mathrm{G}$ (well above the lengthened SCLF limit $B_{\mathrm{d}}=2 \times 10^{14} \mathrm{G}$; such a pulsar was not observed so far), then the actual surface field $B_{\mathrm{s}}$ is well below $B_{\text {cr }} \sim 4 \times 10^{13} \mathrm{G}$, at least in the inner part of the polar cap between $\pm \theta_{\mathrm{v}}=0.0035 \mathrm{rad}$. This "pairforming effective" polar cap is about 2.5 times smaller than the canonical polar cap with radius $\theta_{\mathrm{d}}=0.014 \mathrm{rad}$, and about 7 times smaller than the entire polar cap with radius $\theta_{\mathrm{s}}=0.027 \mathrm{rad}$. Near the last open field lines at polar angles $0.027 \gtrsim|\theta| \gtrsim 0.014$ the actual surface magnetic field $B_{\mathrm{s}}$ is only about 2 times lower than $B_{\mathrm{d}}$, while in a narrow circumpolar area with $|\theta|<\theta_{\mathrm{v}}$ the surface field region $B_{\mathrm{s}}$ can even be more than 10 times weaker than $B_{\mathrm{d}}$. Thus, within our model one can expect a radio-loud HBP with $B_{\mathrm{d}}$ even exceeding $4 \times 10^{14} \mathrm{G}$. However, their radio beams should be much narrower than those expected in normal pulsars, at least a few to several times less than $\left(r_{\mathrm{em}} / R\right)^{1 / 2} P^{-1 / 2}$ degrees (where $r_{\mathrm{em}}$ is the radio emission altitude; Kijak \& Gil 1997, 1998). This would make such sources difficult to detect.

The dotted horizontal line at $B=0.05$ in Fig. 6 corresponds to $B_{\mathrm{s}}=10^{13} \mathrm{G}$ for adopted $B_{\mathrm{d}}=4 \times 10^{14} \mathrm{G}$. This value of the surface magnetic field is believed to be about the lower limit for VG formation (see Gil \& Mitra 2001; Gil et al. 2002). Thus, the shadowed area in Fig. 6 represent a narrow hollow cone above which the VG-driven radio emission cannot occur. A similar hollow cone is marked in Fig. 7, which presents a case similar to that illustrated in Fig. 6, except that the local dipole is shifted off center by $\Delta \theta=0.005$ radians (corresponding to about 0.2 of the actual polar cap radius). The dashed horizontal line at $B=0.4$ corresponds to $B_{\mathrm{s}}=4 \times 10^{13} \mathrm{G}$ and the dotted horizontal line at $B=0.1$ corresponds to $B_{\mathrm{s}}=10^{13} \mathrm{G}$, both calculated for adopted $B_{\mathrm{d}}=2 \times 10^{14} \mathrm{G}$. The polar angles $\theta_{\mathrm{v} 1}$ and $\theta_{\mathrm{v} 2}$ correspond to $-\theta_{\mathrm{v}}$ and $+\theta_{\mathrm{v}}$ in Fig. 6, respectively. Figure 7 demonstrates that the conclusions of our paper do not depend on where the local dipole is placed.

The above arguments strengthen the possibility that some magnetars can also emit observable radio emission (Camilo et al. 2000; Zhang \& Harding 2000b). It is therefore interesting to comment on the apparent proximity of HBP PSRJ $1814-1744$ (with $B_{\mathrm{p}}=1.1 \times 10^{14} \mathrm{G}$ ) and AXP 1 E $2259+586$ (with $B_{\mathrm{p}}=1.2 \times 10^{14} \mathrm{G}$ ) in the $P-\dot{P}$ diagram. In both these cases the inferred surface magnetic field well exceeds the critical value $B_{\mathrm{cr}}$. Within our model, the former object can be radio-loud if the strong local dipole and the global dipole have opposite polarities. The radio quiescence of AXP $1 \mathrm{E} 2259+586$ can be naturally explained if the local dipole is not able to decrease the inferred dipole magnetic field below the photon-splitting death-line. Thus, either the polarities are the same or they are opposite but the local dipole is not strong enough to reduce the dipole surface field below $B_{\mathrm{cr}}$.

In Fig. 8 we show the radii of curvature of actual surface field lines compared with those of purely dipolar field (line 1) as a function of normalized altitude 


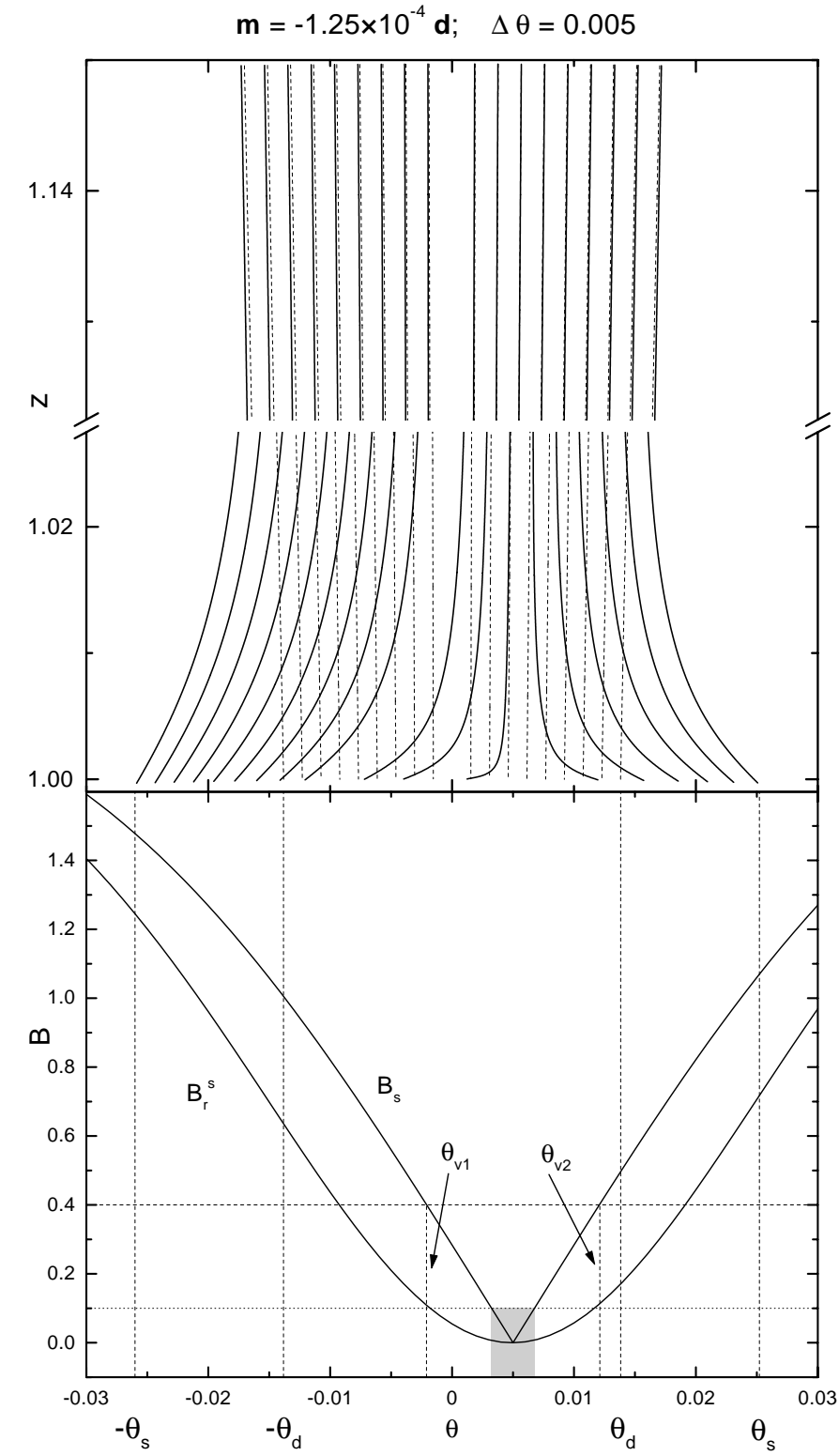

Fig. 7. As in Fig. 6 but with the local dipole shifted off center by $\Delta \theta=0.005$ radians. See also text for explanation.

$z=(r / R) \cos \theta$ above the polar cap. Within the polar gap at $z<1.01$ (within about 100 meters from the surface) the curvature radii for all cases presented in Figs. 2-6 have values of the order of a few hundred meters (see Urpin et al. 1986), suitable for curvature-radiation-driven magnetic pair production $\left(\rho_{\mathrm{c}}=1 / \Re<10^{6} \mathrm{~cm}\right.$, where $\Re$ is the curvature of the field lines).

All model calculations performed in this paper correspond to the axisymmetric case in which one local dipole is placed at the polar cap center (except the case presented in Fig. 7). In a forthcoming paper we will consider a general, non-axisymmetric case, including more local dipoles, each with different orientation with respect to the global dipole. Although this generalization will give a more realistic picture of the actual surface magnetic field, it will not change our conclusions obtained in this paper.

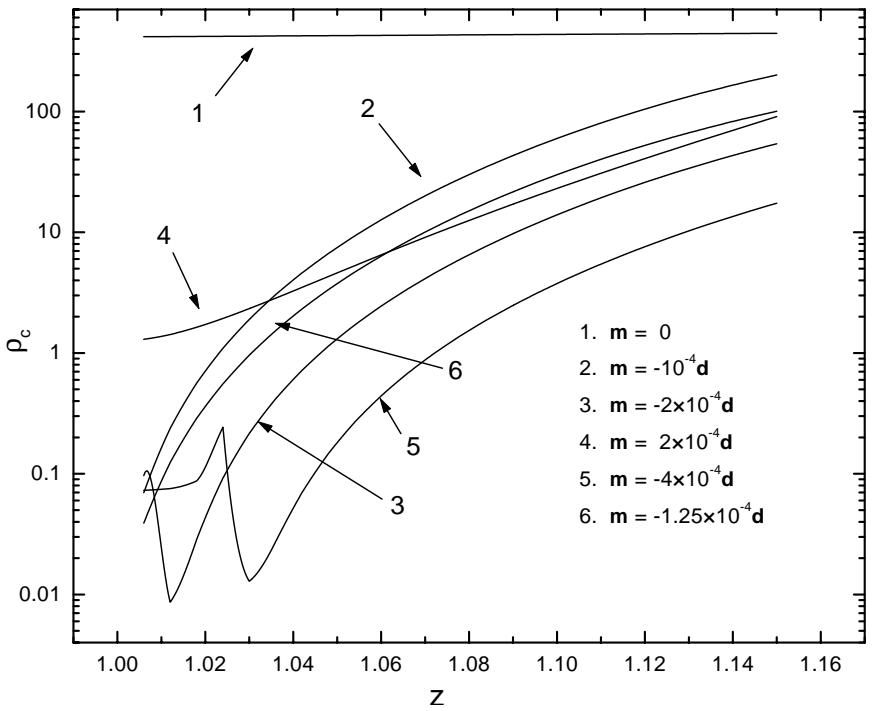

Fig. 8. Radius of curvature $\rho_{\mathrm{c}}$ (in units of $R=10^{6} \mathrm{~cm}$ ) as a function of normalized altitude $z=(r / R) \cdot \cos \theta$ for actual surface magnetic field lines corresponding to four cases presented in Figs. 2-6. For comparison, the radius of curvature of purely dipolar field lines (in pulsar with $P=1 \mathrm{~s}$ ) is shown (line 1).

It should be finally emphasized that although the lengthened SCLF model for HBPs (ZH00) can solve the problem of pair creation in pulsars with surface dipole field exceeding the photon splitting threshold, it does not automatically warrant generation of the coherent radio emission of such HBPs. The problem is that unlike in the non-stationary VG model, where the low altitude radio emission can be generated by means of two-stream instabilities (Asseo \& Melikidze 1998; Melikidze et al. 2000), the stationary SCLF inner accelerator is associated with the high-altitude relativistic maser radiation (e.g. Kazbegi et al. 1991, 1992; Kazbegi et al. 1996). This radiation requires relatively low Lorentz factors $\gamma_{\mathrm{p}} \sim 5 \div 10$ of a dense secondary plasma (e.g. Machabeli \& Usov 1989). It is not clear if such a plasma can be produced within the lengthened SCLF accelerator with delayed pair formation taking place in a purely dipolar magnetic field, either by curvature radiation or by inverse Compton scattering (e.g. Zhang \& Harding 2000b) processes. Moreover, the relativistic maser coherent radio emission requires a relatively weak magnetic field in the generation region. With the surface dipole field $B_{\mathrm{d}} \sim 10^{14} \mathrm{G}$, such a low field may not exist at reasonable altitudes (about $50 \%$ of the light cylinder radius $R_{\mathrm{L}}=c P / 2 \pi$ ) required by the physics of corresponding instabilities (Kazbegi et al. 1991, 1992; Kazbegi et al. 1996). Thus, if one assumes that the radio-loud HBPs are driven by the SCLF lengthened accelerator as proposed by ZH00, they might not be able to generate observable coherent radio emission. This contradiction seems to be a challenge for the lengthened SCLF scenario for HBPs. In our VG-based model the low-altitude $\left(r \ll R_{\mathrm{L}}\right.$ ) radio emission of HBPs is driven by just the same mechanism as the one most probably operating in typical radio pulsars (e.g. soliton curvature 
radiation proposed recently by Melikidze et al. 2000). In fact, the HBPs show apparently normal radio emission, with all properties typical for characteristic pulsar radiation (Camilo et al. 2000).

\section{Appendix A: Surface magnetic field}

In order to model the actual surface magnetic field by superposition of the star-centered global dipole $\boldsymbol{d}$ and the crust-anchored dipole moment $\boldsymbol{m}$, let us consider the general geometrical situation presented in Fig. 1. The resultant surface magnetic field is

$\boldsymbol{B}_{\mathrm{s}}=\boldsymbol{B}_{\mathrm{d}}+\boldsymbol{B}_{\mathrm{m}}$

where

$\boldsymbol{B}_{\mathrm{d}}=\left(\frac{2 d \cos \theta}{r^{3}}, \frac{d \sin \theta}{r^{3}}, 0\right)$,

and

$\boldsymbol{B}_{\mathrm{m}}=\frac{3\left(\boldsymbol{r}-\boldsymbol{r}_{\mathrm{s}}\right)\left(\boldsymbol{m} \cdot\left(\boldsymbol{r}-\boldsymbol{r}_{\mathrm{s}}\right)\right)-\boldsymbol{m}\left|\boldsymbol{r}-\boldsymbol{r}_{\mathrm{s}}\right|^{2}}{\left|\boldsymbol{r}-\boldsymbol{r}_{\mathrm{s}}\right|^{5}}$,

where the spherical components of $\boldsymbol{B}_{\mathrm{m}}$ are explicitly given in Eqs. (A.5). The global magnetic dipole moment $d=$ $(1 / 2) B_{\mathrm{p}} R^{3}$, where $B_{\mathrm{p}}=6.4 \times 10^{19}(P \cdot \dot{P})^{1 / 2} \mathrm{G}$ is the dipole component at the pole derived from the pulsar spin-down rate, and the crust-anchored local dipole moment $m=$ $(1 / 2) B_{\mathrm{m}} \Delta R^{3}$, where $\Delta R \sim 0.05 R<L$ and $L \sim 10^{5} \mathrm{~cm}$ is the characteristic crust dimension $\left(R=10^{6} \mathrm{~cm}\right)$. One can see that a local anomaly can significantly influence the surface magnetic field $\left(B_{\mathrm{m}} \sim B_{\mathrm{d}}\right)$ if $\mathrm{m} / d \gtrsim 10^{-4}$. We use the star-centered spherical coordinates with $z$ axis directed along the global magnetic dipole moment. Thus, $\boldsymbol{r}_{\mathrm{s}}=\left(r_{\mathrm{s}}, \theta_{\mathrm{r}}, \phi_{\mathrm{r}}\right)$ and $\boldsymbol{m}=\left(m, \theta_{\mathrm{m}}, \phi_{\mathrm{m}}\right)$.

The equations of the line of force of the vector field $\boldsymbol{B}\left(B_{\mathrm{r}}, B_{\theta}, B_{\phi}\right)$ have a well known form in spherical co-ordinates

$\frac{\mathrm{d} \theta}{\mathrm{d} r}=\frac{B_{\theta}}{r B_{\mathrm{r}}}, \quad \frac{\mathrm{d} \phi}{\mathrm{d} r}=\frac{B_{\phi}}{r \sin \theta B_{\mathrm{r}}}$

The solution of these equations, with the initial conditions $\theta(r=R)=\theta_{0}$ and $\phi(r=R)=\phi_{0}$ determining a given field line at the surface, describe the parametric equation of the field lines. The magnetic field $\boldsymbol{B}_{\mathrm{m}}$ (Eq. (A.3)), as well as the dipolar magnetic filed $\boldsymbol{B}_{\mathrm{d}}$ (Eq. (A.2)), can be written in terms of spherical components

$$
\begin{aligned}
& B_{\mathrm{r}}^{\mathrm{m}}=-\frac{1}{D^{2.5}}\left(3 T r_{\mathrm{r}}^{\mathrm{s}}-3 T r+D m_{\mathrm{r}}\right) \\
& B_{\theta}^{\mathrm{m}}=-\frac{1}{D^{2.5}}\left(3 T r_{\theta}^{\mathrm{s}}+D m_{\theta}\right) \\
& B_{\phi}^{\mathrm{m}}=-\frac{1}{D^{2.5}}\left(3 T r_{\phi}^{\mathrm{s}}+D m_{\phi}\right)
\end{aligned}
$$

where

$D=r_{\mathrm{s}}^{2}+r^{2}-2 r_{\mathrm{s}} r\left(\sin \theta_{\mathrm{r}} \sin \theta \cos \left(\phi-\phi_{\mathrm{r}}\right)+\cos \theta_{\mathrm{r}} \cos \theta\right)$ and $T=m_{\mathrm{r}} r-\left(m_{\mathrm{r}} r_{\mathrm{r}}^{\mathrm{s}}+m_{\theta} r_{\theta}^{\mathrm{s}}+m_{\phi} r_{\phi}^{\mathrm{s}}\right)$.
According to the geometry presented in Fig. (1), the components of the radius-vector of the origin of the staranchored local dipole moments can be written as

$r_{\mathrm{r}}^{\mathrm{s}}=r_{\mathrm{s}}\left(\sin \theta_{\mathrm{r}} \sin \theta \cos \left(\phi-\phi_{\mathrm{r}}\right)+\cos \theta_{\mathrm{r}} \cos \theta\right)$,

$r_{\theta}^{\mathrm{s}}=r_{\mathrm{s}}\left(\sin \theta_{\mathrm{r}} \cos \theta \cos \left(\phi-\phi_{\mathrm{r}}\right)-\cos \theta_{\mathrm{r}} \sin \theta\right)$,

$r_{\phi}^{\mathrm{s}}=-r_{\mathrm{s}} \sin \theta_{\mathrm{r}} \sin \left(\phi-\phi_{\mathrm{r}}\right)$,

and the components of the local dipole moment are

$m_{\mathrm{r}}=m\left(\sin \theta_{\mathrm{m}} \sin \theta \cos \left(\phi-\phi_{\mathrm{m}}\right)+\cos \theta_{\mathrm{m}} \cos \theta\right)$,

$m_{\theta}=m\left(\sin \theta_{\mathrm{m}} \cos \theta \cos \left(\phi-\phi_{\mathrm{m}}\right)-\cos \theta_{\mathrm{m}} \sin \theta\right)$,

$m_{\phi}=-m \sin \theta_{\mathrm{m}} \sin \left(\phi-\phi_{\mathrm{m}}\right)$.

We now define the boundary of the open field lines by setting $\boldsymbol{B}=\boldsymbol{B}_{\mathrm{d}}$ at an altitude $r_{\mathrm{d}}=50 R$, where the magnetic field should appear like a pure dipole (the numerical results are quite insensitive to the choice of $r_{\mathrm{d}}$ as long as it is few times $R$; in fact, already at $r=5 R$ the ratio $B_{\mathrm{m}} / B_{\mathrm{d}} \sim 10^{-4}$ for $m \approx 10^{-4} d$ ). Then we solve numerically the system of differential equations

$\frac{\mathrm{d} \theta}{\mathrm{d} r}=\frac{B_{\theta}^{\mathrm{d}}+B_{\theta}^{\mathrm{m}}}{r\left(B_{\mathrm{r}}^{\mathrm{d}}+B_{\mathrm{r}}^{\mathrm{m}}\right)} \equiv \Theta_{1}$,

$\frac{\mathrm{d} \phi}{\mathrm{d} r}=\frac{B_{\phi}^{\mathrm{m}}}{r\left(B_{\mathrm{r}}^{\mathrm{d}}+B_{\mathrm{r}}^{m}\right) \sin \theta} \equiv \Phi_{1}$,

obtained from Eq. (A.4) by substitutions $B_{\mathrm{r}}=B_{\mathrm{r}}^{\mathrm{d}}+B_{\mathrm{r}}^{\mathrm{m}}$, $B_{\theta}=B_{\theta}^{\mathrm{d}}+B_{\theta}^{\mathrm{m}}$ and $B_{\phi}=B_{\phi}^{\mathrm{d}}+B_{\phi}^{\mathrm{m}}($ Eqs. (A.2) and (A.5)), with the initial conditions $\boldsymbol{B}_{\mathrm{m}}=0$ defined at $r=50 R$ and trace each field line from altitude $r_{\mathrm{d}}$ (where $\boldsymbol{B}_{\mathrm{m}}\left(r_{\mathrm{d}}, \theta, \phi\right) \equiv$ 0 ) down to the stellar surface $(r=R)$. Here we defined two functions $\Theta_{1}$ and $\Phi_{1}$ to be used later in Eqs. (A.10)(A.12).

The curvature $\Re=1 / \rho_{\mathrm{c}}$ of the field lines (where $\rho_{\mathrm{c}}$ is the radius of curvature presented for various cases in Figs. 2-8) is calculated as

$\Re=\left(\frac{\mathrm{d} s}{\mathrm{~d} r}\right)^{-3}\left|\left(\frac{\mathrm{d}^{2} \boldsymbol{r}}{\mathrm{d} r^{2}} \frac{\mathrm{d} s}{\mathrm{~d} r}-\frac{\mathrm{d} \boldsymbol{r}}{\mathrm{d} r} \frac{\mathrm{d}^{2} s}{\mathrm{~d} r^{2}}\right)\right|$,

where

$\frac{\mathrm{d} s}{\mathrm{~d} r}=\sqrt{\left(1+r^{2} \Theta_{1}^{2}+r^{2} \Phi_{1}^{2} \sin ^{2} \theta\right)}$.

Thus, the curvature can be written in the form

$\Re=\left(S_{1}\right)^{-3}\left(J_{1}^{2}+J_{2}^{2}+J_{3}^{2}\right)^{1 / 2}$,

where

$J_{1}=X_{2} S_{1}-X_{1} S_{2}, \quad J_{2}=Y_{2} S_{1}-Y_{1} S_{2}$,

$J_{3}=Z_{2} S_{1}-Z_{1} S_{2}$

$X_{1}=\sin \theta \cos \phi+r \Theta_{1} \cos \theta \cos \phi-r \Phi_{1} \sin \theta \sin \phi$,

$Y_{1}=\sin \theta \sin \phi+r \Theta_{1} \cos \theta \sin \phi+r \Phi_{1} \sin \theta \cos \phi$,

$Z_{1}=\cos \theta-r \Theta_{1} \sin \theta$,

$X_{2}=\left(2 \Theta_{1}+r \Theta_{2}\right) \cos \theta \cos \phi-\left(2 \Phi_{1}+r \Phi_{2}\right) \sin \theta \sin \phi$ $-r\left(\Theta_{1}^{2}+\Phi_{1}^{2}\right) \sin \theta \cos \phi-2 r \Theta_{1} \Phi_{1} \cos \theta \sin \phi$, 
$Y_{2}=\left(2 \Theta_{1}+r \Theta_{2}\right) \cos \theta \sin \phi+\left(2 \Phi_{1}+r \Phi_{2}\right) \sin \theta \cos \phi$ $-r\left(\Theta_{1}^{2}+\Phi_{1}^{2}\right) \sin \theta \sin \phi+2 r \Theta_{1} \Phi_{1} \cos \theta \cos \phi$,

$Z_{2}=-\Theta_{1} \sin \theta-\Theta_{1} \sin \theta-r \Theta_{2} \sin \theta-r \Theta_{1}^{2} \cos \theta$,

$S_{1}=\sqrt{1+r^{2} \Theta_{1}^{2}+r^{2} \Phi_{1}^{2} \sin ^{2} \theta}$

$S_{2}=S_{1}^{-1}\left(r \Theta_{1}^{2}+r^{2} \Theta_{1} \Theta_{2}+r \Phi_{1}^{2} \sin ^{2} \theta\right.$

$$
\left.+r^{2} \Phi_{1} \Phi_{2} \sin ^{2} \theta+r^{2} \Theta_{1} \Phi_{1}^{2} \sin \theta \cos \theta\right),
$$

$\Theta_{2} \equiv \frac{\mathrm{d} \Theta_{1}}{\mathrm{~d} r}, \quad \Phi_{2} \equiv \frac{\mathrm{d} \Phi_{1}}{\mathrm{~d} r}$

In the two-dimensional case Eq. (A.8) can be simplified to the following form

$\Re=\left(r \frac{\mathrm{d}^{2} \theta}{\mathrm{d} r^{2}}+2 \frac{\mathrm{d} \theta}{\mathrm{d} r}+r^{2}\left(\frac{\mathrm{d} \theta}{\mathrm{d} r}\right)^{3}\right)\left(1+r^{2}\left(\frac{\mathrm{d} \theta}{\mathrm{d} r}\right)^{2}\right)^{-1.5}$.

Acknowledgements. This paper is supported in part by the KBN Grant 2 P03D 00819 of the Polish State Committee for Scientific Research. We are grateful to Dr. U. Geppert for very helpful discussions. We also thank E. Gil and G. Melikidze Jr. for technical help. DM would like to thank the Institute of Astronomy, University of Zielona Góra, for support and hospitality during his visit to the institute, where this and the accompanying paper were started.

\section{References}

Adler, S. L., Bahcall, J. N., Callan, C. G., \& Rosenbluth, M. N. 1970, Phys. Rev. Lett., 25, 1061

Arons, J. 1981, ApJ, 248, 1099

Arons, J. 1993, ApJ, 408, 160

Arons, J., \& Sharleman, E. T. 1979, ApJ, 231, 854

Asseo, E., \& Melikidze, G. 1998, MNRAS, 301, 59

Baring, G. M. 2001, to appear in Proc Tonantzintla Workshop, The nature of Unidentified Galactic High-Energy Gamma-Ray Sources, ed. A. Carraminana, et al. (Kluwer Academic, Dordrecht) [astro-ph/0106161]

Baring, G. M., \& Harding, A. K. 1998, ApJ, 507, L55

Baring, G. M., \& Harding, A. K. 2001, ApJ, 547, 929

Becker, W., \& Trümper, J. 1997, A\&A, 326, 682

Bhattacharya, D., \& Shukre, C. S. 1985, JApA, 6, 233

Bialynicka-Birula, Z., \& Bialynicki-Birula, I. 1970, Phys. Rev., 2,2341

Blandford, R. D., Applegate, J. H., \& Hernquist, L. 1983, MNRAS, 204, 1025

Bulik, T., Meszaros, P., Woo, J., Nagase, F., \& Makishima, K. 1992, ApJ, 395, 564

Bulik, T., Riffert, H., Meszaros, P., Makishima, S., \& Mihara, T. 1995, ApJ, 444, 405

Camilo, F., Kaspi, V. M., Lyne, A. G., et al. 2000, ApJ, 541, 367

Chen, K., \& Ruderman, M. A. 1993, ApJ, 402, 264

Cheng, K., \& Ruderman, M. A. 1977, ApJ, 214, 598

Cheng, K., \& Ruderman, M. A. 1980, ApJ, 235, 576

Cheng, K. S., Gil, J., \& Zhang, L. 1998, ApJ, 493, L35

Cheng, K. S., \& Zhang, L. 1999, ApJ, 515, 337

Cordes, J. M. 1978, ApJ, 222, 1006

Cordes, J. M. 1992, in IAU Coll. 128, ed. T. H. Hankins, J. M. Rankin, \& J. A. Gil Zielona (Gora: Pedagogical Univ. Press), 253

Cordes, J. M. 2001, Nature, 409, 296

Deshpande, A. A., \& Rankin, J. M. 1999, ApJ, 524, 1008
Deshpande, A. A., \& Rankin, J. M. 2001, MNRAS, 322, 438

Duncan, R., \& Thompson, C. 1992, ApJ, 392, L9

Fillipenko, A. V., \& Radhakrishnan, V. 1982, ApJ, 263, 828

Geppert, U., \& Urpin, V. 1994, MNRAS, 271, 490

Gil, J., \& Sendyk, M. 2000, ApJ, 541, 351

Gil, J., \& Mitra, D. 2001, ApJ, 550, 383

Gil, J., Melikidze, G. I., \& Mitra, D. 2002, A\&A, 388, 246

Goldreich, P., \& Julian, W. H. 1969, ApJ, 157, 869

Gotthelf, E. V., Vasisht, G., Boylan-Kolchin, M., \& Torii, A. 2000, ApJ, 542, 37L

Hillebrandt, W., \& Müller, E. 1976, ApJ, 207, 589

Kaspi, N. M., Manchester, R. N., Johnston, S., et al. 1996, AJ, 111(5), 2029

Kazbegi, A. Z., Machabeli, G. Z., \& Melikidze, G. I. 1991, MNRAS, 253, 377

Kazbegi, A. Z., Machabeli, G. Z., \& Melikidze, G. I. 1992, in IAU Coll. 128, ed. T. H. Hankins, J. M. Rankin, \& J. A. Gil Zielona (Gora Pedagogical Univ. Press), 232

Kazbegi, A. Z., Machabeli, G. Z., Melikidze, G. I., et al. 1996, A\&A, 309, 515

Kijak, J. 2001, MNRAS, 323, 537

Kijak, J., \& Gil, J. 1997, MNRAS, 288, 631

Kijak, J., \& Gil, J. 1998, MNRAS, 299, 855

Kössl, D., Wolff, R. G., Müller, E., \& Hillebrandt, W. 1988, A\&A, 205, 347

Krolik, J. W. 1991, ApJ, 373, L69

Machabeli, G. Z., \& Usov, V. V. 1989, Sov. Astron. Lett., 15(5), 393

Melikidze, G. I, Gil, J., \& Pataraya, A. D. 2000, ApJ, 544, 1081

Mitra, D., Konar, S., \& Bhattacharaya, D. 1999, MNRAS, 307, 459

Murakami, T., Kubo, S., Shibazaki, N., et al. 1999, ApJ, 510, L119

Page, D., \& Sarmiento, A. 1996, ApJ, 473, 1067

Pirovaroff, M. J., Kaspi, V. M., \& Camilo, F. 2000, ApJ, 535, 379

Radhakrishnan, V., \& Cooke, D. J., 1969, ApL, 3, 225

Rudak, B., \& Dyks J. 1999, MNRAS, 303, 477

Ruderman, M. A., \& Sutherland, P. G. 1975, ApJ, 196, 51

Ruderman, M. A. 1991, ApJ, 366, 261

Shapiro, S. L., \& Teukolsky, S. A. 1983, Black Holes, White Dwarfs and Neutron Stars: The Physics of Compact Objects (New York: Wiley)

Sharleman E. T., Arons J., \& Fawley W. M. 1978, ApJ, 222, 297

Sturrock, P. A. 1971, ApJ, 164, 529

Tauris, T. M., \& Konar, S. 2001, A\&A, 376, 543

Thompson, C., \& Duncan, R. C. 1995, MNRAS, 275, 255

Thompson, C., \& Duncan, R. C. 1996, ApJ, 473, 322

Urpin, V. A., Levshakov, S. A., \& Yakovlev, D. G. 1986, MNRAS, 219, 703

Usov, V. V. 1987, ApJ, 481, L107

Usov, V. V., \& Melrose, D. B. 1995, Austr. J. Phys., 48, 571

Usov, V. V., \& Melrose, D. B. 1996, ApJ, 463, 306

Woltjer, L. 1964, ApJ, 140, 1309

Zhang, B., \& Harding, A. K. 2000a, ApJ, 535, L51 (ZH00)

Zhang, B., \& Harding, A. K. 2000b, ApJ, 532, 1150

Zhang, B., \& Harding, A. K. 2001, Proc. of Soft Gamma Repeaters: The Rome 2000 Mini-Workshop, ed. M. Feroci, \& S. Mereghetti, Mem. S. A. It. (ZH01) [astro-ph/0102097]

Zhang, B. 2001, ApJ, 562, L59 